\title{
Light Harvesting at Oblique Incidence Decoupled from Transmission in Organic Solar Cells Exhibiting 9.8\% Efficiency and $50 \%$ Visible Light Transparency
}

\author{
Quan Liu, Luis G. Gerling, Francisco Bernal-Texca, Johann Toudert, Tengfei Li, \\ Xiaowei Zhan,* and Jordi Martorell**
}

For many years, it has been recognized that potential organic photovoltaic cells must be integrated into elements requiring high transparency. In most of such elements, sunlight is likely to be incident at large angles. Here it is demonstrated that light transmission can be largely decoupled from harvesting by optically tailoring an infrared shifted nonfullerene acceptor based organic cell architecture. A $9.67 \%$ power conversion efficiency at $50^{\circ}$ incidence is achieved together with an average visual transmission above $50 \%$ at normal incidence. The deconstruction of a ID nanophotonic structure is implemented to conclude that just two $\lambda / 4$ thick layers are essential to reach, for a wide incidence angle range, a higher than $50 \%$ efficiency increase relative to the standard configuration reference. In an outdoor measurement of vertically positioned $50 \%$ visible transparent cells, it is demonstrated that $9.80 \%$ of sunlight energy can be converted into electricity during the course of 1 day.

acceptors (NFAs) with an infrared shifted absorption yielded impressive cell performances for cells with different levels of transparency. ${ }^{[5-19]}$ However, the ultimate route to obtain a highly efficient energy conversion in highly transparent cells may deviate significantly from the one followed to reach the best opaque solar cells, which essentially targets a maximization of the power conversion efficiency (PCE) at normal incidence. The final architecture for an optimal transparent solar cell may be strongly linked to where on the planet earth such transparent light harvesting device is located, its azimuth and inclination, and also to the application that may be given to such device in addition to energy conversion. For a vertically positioned semitransparent light harvesting

\section{Introduction}

The absorption characteristics of the organic photovoltaic (OPV) materials make them ideal candidates to achieve visibly transparent devices that would be able to compete with a Si-based technology in the conversion of light to electricity. ${ }^{[1-4]}$ Recent advances in the synthesis of novel nonfullerene

Dr. Q. Liu, Dr. L. G. Gerling, F. Bernal-Texca, Dr. J. Toudert,

Prof. J. Martorell

ICFO-Institut de Ciències Fotòniques

The Barcelona Institute of Science and Technology

Castelldefels, Barcelona 08860, Spain

E-mail: jordi.martorell@icfo.eu

T. Li, Prof. X. Zhan

Department of Materials Science and Engineering

College of Engineering

Key Laboratory of Polymer Chemistry and Physics of Ministry

of Education

Peking University

Beijing 100871, China

E-mail: xwzhan@pku.edu.cn

Prof. J. Martorell

Departament de Física

Universitat Politècnica de Catalunya

Terrassa 08222, Spain

The ORCID identification number(s) for the author(s) of this article can be found under https://doi.org/10.1002/aenm.201904196.

DOI: 10.1002/aenm.201904196 window, vision through the device should remain unaltered at angles relatively close to normal incidence $\left(\approx 0-50^{\circ}\right)$ while at large angles sunlight harvesting should be maximized, as shown schematically in Figure 1a.

\section{Results and Discussion}

\subsection{Transparent Cell Architecture Design}

A blend mixing a narrow bandgap donor polymer as PTB7-Th and an NFA acceptor as FOIC has been shown to efficiently utilize near infrared (NIR) solar irradiation making them an ideal material to consider semitransparent cells. ${ }^{[9]}$ Departing from such blend, we introduced $\mathrm{PC}_{71} \mathrm{BM}$ fullerene acceptor in the proportion specified in the experimental section to slightly increase the device fill factor $(\mathrm{FF})$ and open-circuit voltage $\left(V_{\mathrm{OC}}\right)$ without any major effect in the short-circuit current or visible transmission. The final material composition with an absorption band peaking at $820 \mathrm{~nm}$ is the ideal one to use in semitransparent cells provided such band may be optically shifted or broadened to reach the ultimate balance between energy conversion and transparency.

Without introducing any further changes in the blend nanomorphology, we considered the incorporation into such organic cell of a 1D nanophotonic structure (1D-NPS) to effectively harvest sunlight when the cell is in a vertical position. Such 
a

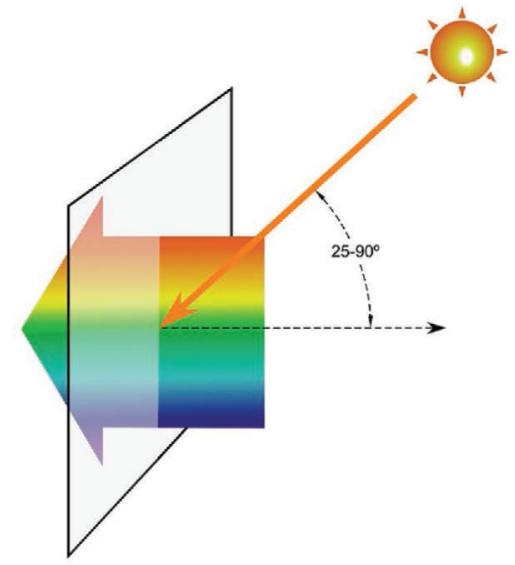

C

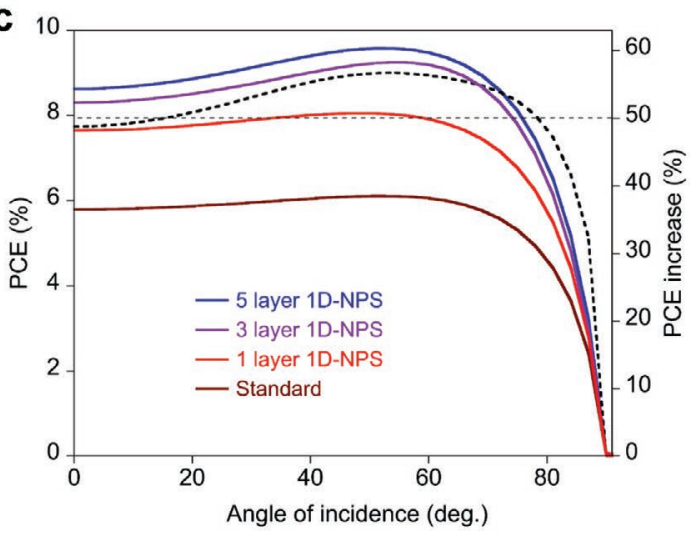

b

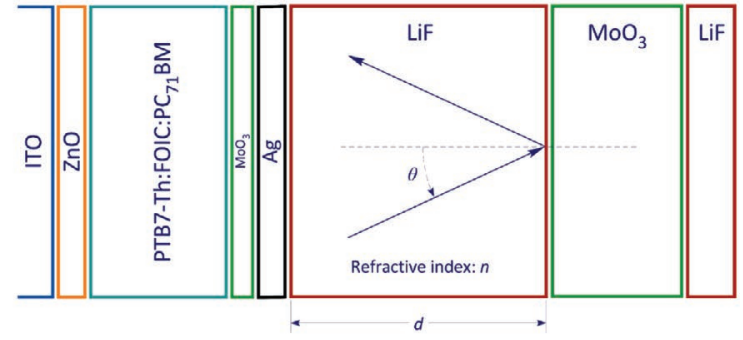

d

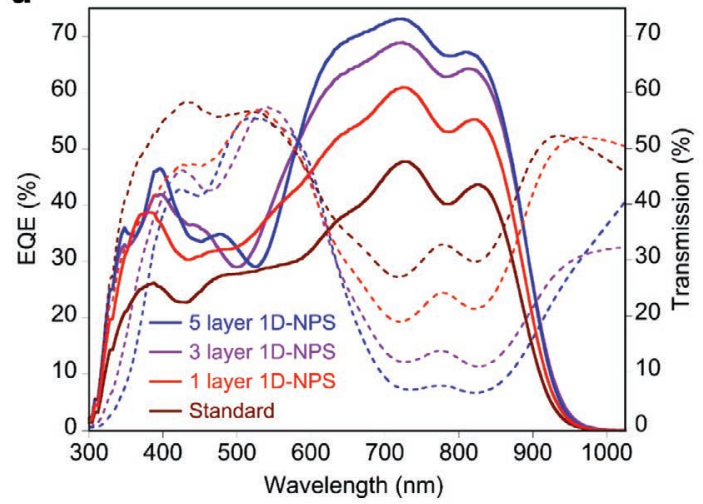

Figure 1. Design of the 1D-NPS for an optimal energy conversion in transparent cells. a) Transparent light harvesting window. At parallel $41^{\circ}$ direct sunlight is harvested at angles ranging from $25^{\circ}$ to $90^{\circ}$. b) Schematic cell architecture including a three-layer 1D-NPS. c) Power conversion efficiency (PCE, left axis) as a function of the light angle of incidence. The PCE increase of a five-layer 1D-NPS relative to the standard architecture is shown in the right axis as a dotted line. d) External quantum efficiency (EQE) at the angle where the PCE is maximum (left axis) with the corresponding light transmission at normal incidence (right axis). All four cells considered in c,d) share the basic architecture soda-lime glass substrate/ITO (135 $\mathrm{nm}$ )/ $\mathrm{ZnO}-\mathrm{SC}(10 \mathrm{~nm}) / \mathrm{AL} / \mathrm{MoO}_{3}(5 \mathrm{~nm}) / \mathrm{Ag}(9 \mathrm{~nm})$ where AL indicates the same PTB7-Th:FOIC:PC $\mathrm{BM}$ active layer blend. ${ }^{[29]}$ The AL thickness is $50,71,85$, and 87 for the standard, one-, three-, and five-layer 1D-NPS configurations, respectively. The standard, one-, three-, and five-layer ID-NPS configurations are capped with LiF $(30 \mathrm{~nm})$, LiF $(262 \mathrm{~nm}), \mathrm{LiF}(149 \mathrm{~nm}) / \mathrm{MoO}_{3}(78 \mathrm{~nm}) / \mathrm{LiF}(43 \mathrm{~nm})$, and LiF $(152 \mathrm{~nm}) / \mathrm{MoO}_{3}(70 \mathrm{~nm}) / \mathrm{LiF}(137 \mathrm{~nm}) / \mathrm{MoO} 3(95 \mathrm{~nm}) /$ LiF $(60 \mathrm{~nm})$, respectively. All the 1D-NPS cells incorporate a LiF $(120 \mathrm{~nm})$ antireflection coating on the opposite side of the substrates.

structure, alternating low and high refractive index nanothick layers, is shown schematically in Figure $1 \mathrm{~b}$. To avoid introducing any relevant alteration to parameters which, in the end, will largely determine the electrical performance of an organic solar cell, we limited the study to 1D-NPSs incorporating dielectric materials that can be deposited using mild conditions in high vacuum evaporation. ${ }^{[20-26]}$ To find the optimal configuration an often used procedure is based on an inverse design ${ }^{[27-31]}$ taking into account all the specificities of light harvesting. In addition to standard considerations such as the light source, the absorption wavelength dependence, and the human eye response to light, we should incorporate in the design a vertical cell positioning, as well as an intensity and angular-dependent irradiation. As a consequence, the cell performance cannot be solely evaluated on PCE and light transmission as it was done in the past, ${ }^{[32,33]}$ but must primarily consider the efficiency in collecting and converting light into electricity, defined as the ratio of the electrical energy obtained from the photovoltaic device in relation to the received sunlight energy during the course of 1 day. Throughout the text we will use the acronym ECE which stands for energy conversion efficiency.
To compute the optimal transparent cell architecture, we used the transfer matrix full wave vector method incorporating an angular and intensity dependent sun irradiation, and sought a solution following an inverse design approach as described in Section 1 of the Supporting Information and ref. [34]. In such computation, the sun irradiation was estimated using equation S6 (Supporting Information). We also took into account that outdoor energy harvesting experimental measurements were performed at parallel $\approx 41^{\circ}$ on sunny days during or close to the spring equinox. The vertical inclination and azimuthal orientation of the cell was incorporated in the computation, too. When the target in the inverse design was a cell with an average visual transmission (AVT) at normal incidence higher than 50\% and providing the maximum ECE at the end of the day, the optimal cell architecture found was one incorporating a five-layer 1D-NPS behind a $9 \mathrm{~nm}$ thick back Ag metal contact. As seen in Figure 1c, incorporating such 1D-NPS increases the PCE relative to the standard architecture transparent cell by more than $50 \%$ for almost the entire light incidence angular range. Increasing the number of layers from 1 to 5 in the 1D-NPS tends to increase the external quantum efficiency (EQE) in the 
NIR range as well as broaden such EQE toward the visible part of the spectrum, as can be seen in Figure 1d. This broadening would not imply any reduction in the performance of the cell as a transparent device provided light harvesting is largely decoupled from visible transmission at normal incidence as can be seen in Figure 1d.

At first sight, inspection of the thicknesses in the fivelayer 1D-NPS (given in the caption of Figure 1) does not show any apparent relationship among them, which may lead one to conclude that a disordered 1D-NPS is needed for an optimal cell performance. In what follows we deconstruct such inverse design to bring new insight into the optical tailoring brought by the 1D-NPS. In principle, such inverse design should lead to a layered structure enhancing reflectivity at the NIR with wavelengths ranging approximately from 600 to $900 \mathrm{~nm}$. What is somehow surprising is that the phase shift

$2 \frac{2 \pi}{\lambda} n d \cos \theta+\pi$

introduced to a reflected wave propagating at an angle $\theta$ relative to the normal by any given layer of thickness $d$ and refractive index $n$, is $2 \pi$ (see Figure S3 in the Supporting Information), only for the first two and four layers in the three- and fivelayer 1D-NPS, respectively. In fact, the largest contribution to the $50 \%$ enhancement in the EQE is provided by the first two layers adjacent to the back metal contact in both structures. At normal incidence (Figure S3a, Supporting Information), both such layers correspond to a $\lambda / 4$ thickness but separated by about $200 \mathrm{~nm}$. For the five-layer 1D-NPS the thicknesses of the third and fourth layers are also $\lambda / 4$ but at wavelengths significantly different from the ones corresponding to the first and second layers. These latter layers provide a fine optical tuning, slightly increasing energy collection in the UV and NIR part of the spectrum and at all angles as seen in Figure 1d,c respectively. At $50^{\circ}$ incidence (Figure S3b, Supporting Information), the separation among the four different wavelengths tends to narrow down. In both structures, the last layer is a very thin layer with almost the sole purpose to reduce the refractive index mismatch with air. As for the one-layer 1D-NPS, the thickness is $\lambda / 2$ instead because of an additional $\pi$ phase shift at the 1D-NPS back interface where the index changes from high to low. There is a clear EQE sharpening in the 600 to $900 \mathrm{~nm}$ range when changing from a one- to a three-layer 1D-NPS, as a result of a partial coherent superposition of the reflectivity provided by one additional interface in the threelayer case. However, such sharpening is significantly lower when any additional $\lambda / 4$ layer is incorporated. In summary, only two of such $\lambda / 4$ layers at different wavelengths are essentially needed. This is in sharp contrast to a Bragg reflection that would include many of such layers resonating at a single wavelength. The fact that only two layers are strictly needed also contrasts with disordered configurations considered in the past where a larger number of dielectric layers were used. It is also worth noticing that the small number of $\lambda / 4$ layers needed to achieve an optimal performance make the final 1D-NPS design largely insensitive to the specific conditions initially set as, for instance, the cell orientation relative to the incident sun rays. In other words, the 1D-NPS is essential to efficiently capture light at large angles. However, the final architecture obtained designing the 1D-NPS when considering light at normal incidence would exhibit minor differences relative to the one obtained when considering light incident with a broad angular range.

\subsection{Implementation of 1D-NPS Cells}

The largest ECE with an AVT above $50 \%$ can be reached by implementing the five-layer 1D-NPS considered above. This requires the fabrication of a semitransparent $\mathrm{Ag}$ electrode on top of the cell structure with a thickness of $9 \mathrm{~nm}$. Such thickness is below the standard one for such type of top electrodes which is typically above $10 \mathrm{~nm} \cdot{ }^{[7,9,35,36]}$ To improve the wetting of silver on top of the $\mathrm{MoO}_{3}$ hole transporting layer while avoiding the formation of island-like clusters that lead to a metal thin film with low transmission and low conductivity, ${ }^{[37,38]}$ we deposited an ultrathin $\mathrm{Au}$ seed layer $(\approx 1 \mathrm{~nm})$ prior to the Ag evaporation on a cold substrate. ${ }^{[39-41]}$ Further details on the novel procedure we implemented to obtain semitransparent Ag top electrodes as thin as $8 \mathrm{~nm}$ (see scanning electron microscope images in Figure S4, Supporting Information), can be found in Experimental section. To ensure a minimum reduction in the device FF compared to opaque electrodes, the Ag thickness in transparent cells should be above $9 \mathrm{~nm}$ (Figure S5, Supporting Information). After the top electrode deposition, the 1D-NPS was fabricated by the alternated high vacuum evaporation of $\mathrm{LiF}$ and $\mathrm{MoO}_{3}$ layers. As indicated in the previous section, these materials were chosen because the deposition conditions are mild enough to not lead to a significant reduction in the device electrical properties. Details on the deposition procedure followed can be found in the Experimental section, too.

To confirm the capacity of the five-layer 1D-NPS cell to effectively harvest light at large incidence angles, we measured the EQE of such cells at $0^{\circ}$ and $50^{\circ}$, as shown in Figure 2a. Note that the NIR band of the EQE broadens toward the blue part of the spectrum by more than $55 \mathrm{~nm}$ when the cell is rotated by $50^{\circ}$ with respect to light incidence. As can be seen in Figure $2 \mathrm{~b}$, this is corresponded with an increase in the PCE, which at $50^{\circ}$ reaches a maximum value of $9.67 \%$ compared to the maximum reached at normal incidence which was $8.66 \%$ as indicated in Table 1. In contrast, the EQE as well as the current densityvoltage $(J-V)$ curves of a standard configuration transparent organic solar do not exhibit any significant change when light incidence changes from $0^{\circ}$ to $50^{\circ}$. Note that the standard configuration cell we used as a reference device exhibits a performance which is better than performances reported in the recent literature that considered organic cells with a $50 \%$ transparency or above (see Table S1, Supporting Information). In addition, when the light harvesting capacity is weighted with the AVT (PCE.AVT product), the cells including the five-layer 1D-NPS almost double the performance of such reference cells (Figure S7, Supporting Information). When comparing it to the most recent report on $50 \%$ transparency OPV cells, such relative gain becomes larger than $100 \%$ (green arrow in Figure S7, Supporting Information).

Although for both the five-layer 1-D NPS and standard cells the AVT is slightly above $50 \%$, it is clear by inspection of Figure $2 \mathrm{c}$ 

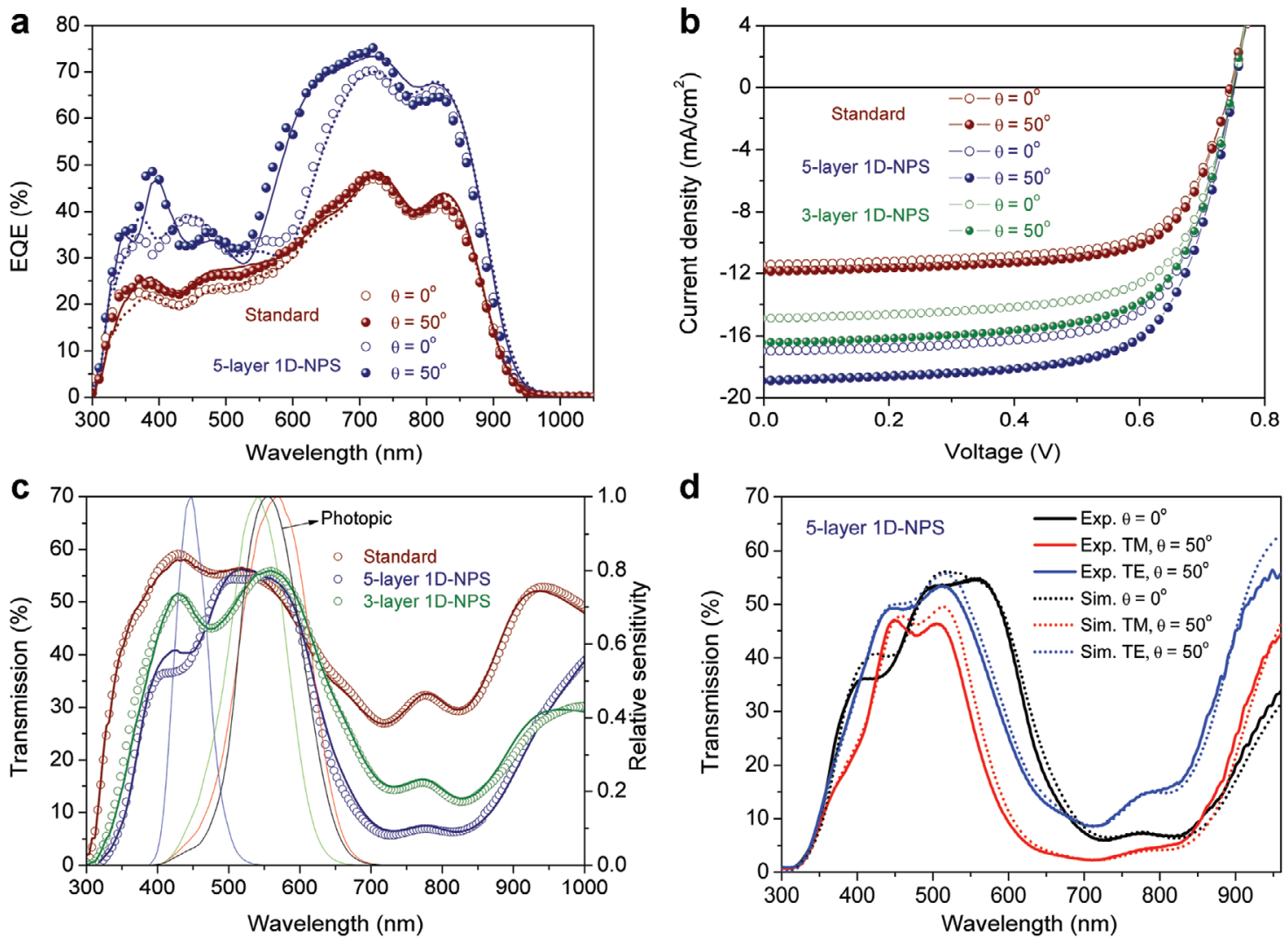

Figure 2. Cell conversion efficiency and transparency. a) Experimental (circles) and simulated (lines) EQEs for the standard (dark red) and five-layer ID-NPS (blue) cells at normal (empty circles and dotted lines) and at $50^{\circ}$ (solid circles and solid lines) incidence. b) Measured J-V characteristics of the standard (dark red), five-layer 1D-NPS (blue) and three-layer 1D-NPS (green) cells at normal (empty circles) and $50^{\circ}$ (solid circles) incidence under AM1.5G 1-sun illumination. c) Experimental (empty circles) and simulated (solid lines) light transmission spectra at normal incidence (left axis) for the standard (dark red), five-layer 1D-NPS (blue) and three-layer 1D-NPS (green) cells. The normalized S (blue thin line), M (green thin line), L (red thin line) cone sensitivities and photopic curves (black thin line) are also depicted in the right axis. d) Experimental (solid) and simulated (dotted) TM (red) and TE (blue) light transmissions at $50^{\circ}$ and normal (black) incidence for the five-layer ID-NPS cell. All cells considered in a-d) share the basic architecture soda-lime glass substrate/ITO $(135 \mathrm{~nm}) / \mathrm{ZnO}-\mathrm{SG}(10 \mathrm{~nm}) / \mathrm{AL} / \mathrm{MoO}_{3}(5 \mathrm{~nm}) / \mathrm{Ag}(9 \mathrm{~nm})$, where AL is the same PTB7-Th:FOIC:PC $\mathrm{BM}$ active layer blend with a thickness of 50, 72, and $86 \mathrm{~nm}$ for the standard, three-, and five-layer 1D-NPS configurations, respectively. The standard, three-, and five-layer ID-NPS configurations are capped with LiF $(30 \mathrm{~nm})$, LiF $(140 \mathrm{~nm}) / \mathrm{MoO}_{3}(98 \mathrm{~nm}) / \mathrm{LiF}(40 \mathrm{~nm})$ and LiF $(150 \mathrm{~nm}) / \mathrm{MoO} 3(70 \mathrm{~nm}) / \mathrm{LiF}(132 \mathrm{~nm}) /$ $\mathrm{MoO}_{3}(97 \mathrm{~nm}) / \mathrm{LiF}(56 \mathrm{~nm})$, respectively. All 1D-NPS cells incorporate a LiF $(120 \mathrm{~nm})$ antireflection coating on the opposite side of the substrate. Note that the three-layer 1D-NPS cell was optimized for a neutral color transmission as opposed to the one considered in Figure 1, which was optimized for an AVT larger than 50\% only. The differences between the five-layer 1-D NPS from the current figure and the one from Figure 1 must be attributed to experimental limitations in exactly reproducing layer thicknesses.

Table 1. Photovoltaic parameters for the fabricated solar cells under AM1.5G 1-sun solar illumination.

\begin{tabular}{|c|c|c|c|c|c|c|c|c|}
\hline Device & $\begin{array}{c}\mathrm{AL} \\
\text { thickness }\end{array}$ & $\begin{array}{l}\mathrm{LiF} / \mathrm{MoO}_{3} / \mathrm{LiF} / \\
\mathrm{MoO}_{3} / \mathrm{LiF}[\mathrm{nm}]\end{array}$ & $\begin{array}{c}\text { Angle of } \\
\text { incidence }\end{array}$ & $\begin{array}{l}J_{\mathrm{Sc}}\left(J_{\mathrm{EQE}}\right)^{\mathrm{a})} \\
{\left[\mathrm{mA} \mathrm{cm}{ }^{-2}\right]}\end{array}$ & $\begin{array}{l}V_{\text {OC }} \\
{[\mathrm{V}]}\end{array}$ & $\begin{array}{l}\mathrm{FF} \\
{[\%]}\end{array}$ & $\begin{array}{c}\mathrm{PCE}^{\mathrm{b})} \\
{[\%]}\end{array}$ & $\begin{array}{c}\left.\mathrm{AVT}^{\mathrm{c}}\right) \\
{[\%]}\end{array}$ \\
\hline \multirow[t]{2}{*}{ Standard } & $50 \mathrm{~nm}$ & $30 / 0 / 0 / 0 / 0$ & $\theta=0^{\circ}$ & $11.34 \pm 0.15(11.30)$ & $0.744 \pm 0.002$ & $68.1 \pm 1.0$ & $5.75 \pm 0.07(5.82)$ & 50.92 \\
\hline & & & $\theta=50^{\circ}$ & $11.75 \pm 0.21$ (11.69) & $0.744 \pm 0.002$ & $68.2 \pm 1.1$ & $6.02 \pm 0.08(6.11)$ & \\
\hline \multirow[t]{2}{*}{ Five-layer 1D-NPS } & $86 \mathrm{~nm}$ & $150 / 70 / 132 / 97 / 56$ & $\theta=0^{\circ}$ & $16.69 \pm 0.22(16.64)$ & $0.746 \pm 0.003$ & $66.8 \pm 0.8$ & $8.32 \pm 0.18(8.66)$ & 50.04 \\
\hline & & & $\theta=50^{\circ}$ & $18.73 \pm 0.26(18.54)$ & $0.751 \pm 0.002$ & $66.8 \pm 0.7$ & $9.40 \pm 0.16(9.67)$ & \\
\hline \multirow[t]{2}{*}{ Three-layer ID-NPS (neutral color) } & $72 \mathrm{~nm}$ & $140 / 98 / 40 / 0 / 0$ & $\theta=0^{\circ}$ & $14.72 \pm 0.17(14.62)$ & $0.749 \pm 0.002$ & $66.7 \pm 1.1$ & $7.36 \pm 0.16(7.56)$ & 51.09 \\
\hline & & & $\theta=50^{\circ}$ & $16.43 \pm 0.25(15.98)$ & $0.744 \pm 0.003$ & $66.8 \pm 1.0$ & $8.16 \pm 0.12(8.34)$ & \\
\hline Opaque (optimal) & $105 \mathrm{~nm}$ & - & $\theta=0^{\circ}$ & $23.83 \pm 0.33(23.10)$ & $0.753 \pm 0.005$ & $66.5 \pm 1.3$ & $11.95 \pm 0.28(12.32)$ & 0 \\
\hline
\end{tabular}

a) Calculated from experimental EQE spectra; $\left.{ }^{b}\right)$ The average values and standard deviations are obtained from over 15 devices and the best PCE values are shown in paren theses; ${ }^{c)}$ AVT is calculated from $\int T(\lambda) V(\lambda) I(\lambda) \mathrm{d} \lambda / \int V(\lambda) I(\lambda) \mathrm{d} \lambda$, where $T(\lambda)$ is experimental transmission, $V(\lambda)$ is photopic curve, and $I(\lambda)$ is the AM1.5G spectrum (see Section S4, Supporting Information). 


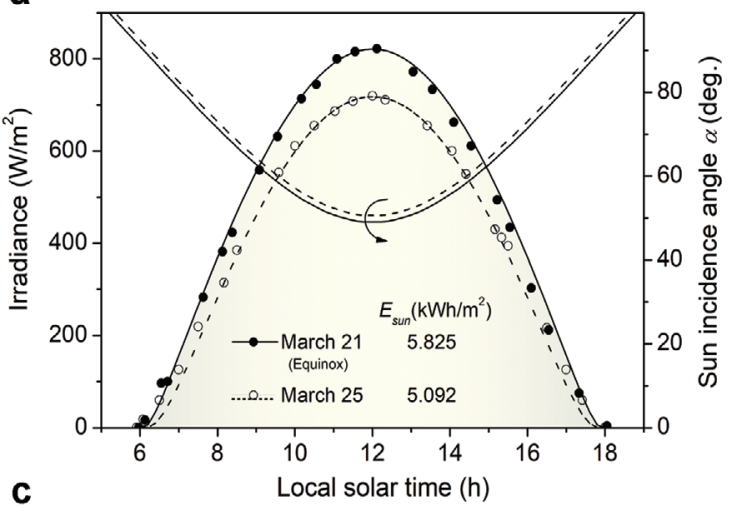

C

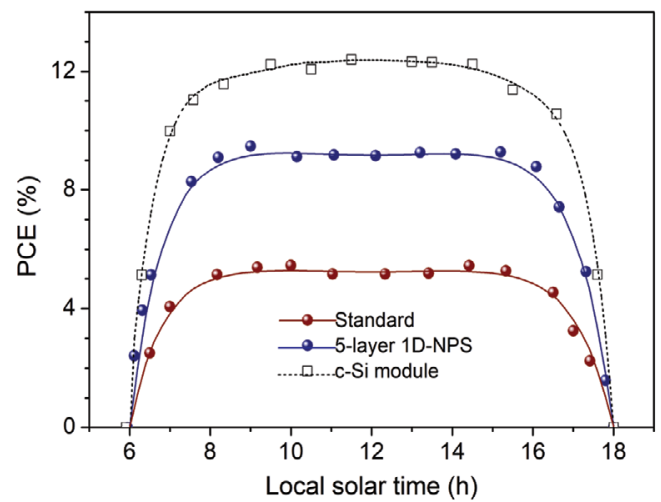

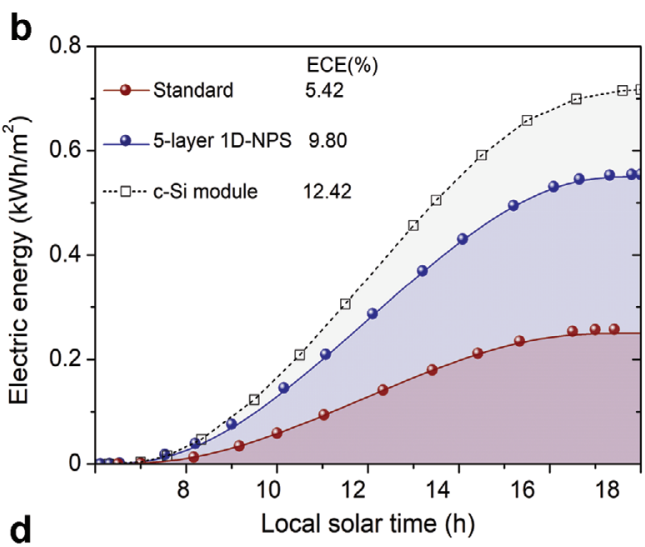

d

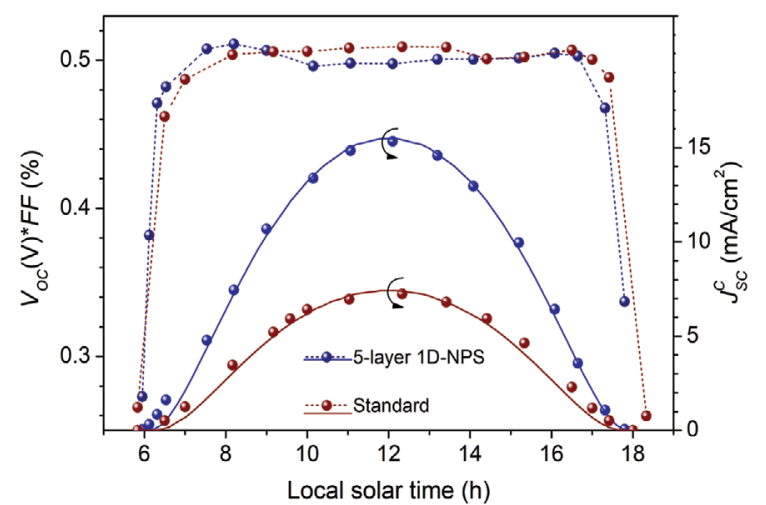

Figure 3. Outdoor measurements of energy conversion during the equinox. a) Experimental (circles) and modeled (lines) sun irradiance (left axis) and sun rays angle of incidence (right axis) on a vertical cell facing south for two different days: March 212019 (solid circles, solid lines) and March 252019 (empty circles, dashed lines). The angle of sun rays' incidence is given relative to the normal to the cell surface. The five-layer 1D-NPS and mc-Si module were measured on March 21 and the standard cell on March 25. The area under the curves corresponds to the total sun radiation used to determine the ECE. b) Accumulated electric energy $\left(\mathrm{kWh} \mathrm{m}^{-2}\right)$ for the standard and five-layer 1D-NPS cells and the mc-Si module. c) PCE of the fabricated cells and $\mathrm{mc}$-Si module during the course of the day, using the modeled irradiance from a) as the input power. d) Experimental $V_{\mathrm{O} C} \cdot \mathrm{FF}$ product (left axis) and $J_{\mathrm{sc}}^{\mathrm{c}}$ relative to the cell area (right axis). All measurements were performed in a south-oriented vertical position during clear sky days. Cell values represent a 16-device average while mc-Si module are single measurements (see Tables S2, S3, and S4 of the Supporting Information for listed data). Solid or dashed lines are model predictions whereas dotted lines are guides to the eye.

that the standard configuration cell would provide a closer to a neutral color transmission given that transmission in the blue as well as the red portions of the spectrum is less affected by such solar cell. To achieve a high efficiency neutral color transmission solar cell, we incorporated in the inverse design the condition that, in addition to an AVT above $50 \%$, the average transmission for the blue, red, and green cone sensitivities to be above 50\%, too (see Figure S8 in the Supporting Information). In that case, the inverse design yielded a three-layer 1D-NPS cell that when fabricated exhibited a maximum PCE of $8.34 \%$, more than 2 percentage points higher than the PCE of the best standard configuration cell (see $J-V$ curve in Figure $2 \mathrm{~b}$ and EQE curve in Figure S9 in the Supporting Information). On the other hand, as can be seen in Figure 2c and Figure S10 (Supporting Information), the transmission profile achieved for the three-layer 1D-NPS cell is very similar to the one from the standard configuration.

In an angular dependent optimization of an organic solar cell, one may have to consider additional adjustments to account for a possible birefringence due to a preferred orientation of the polymer or molecules within the active layer. ${ }^{[42]}$ To evaluate potential deviations in the refractive index, we measured the transverse magnetic (TM) and transverse electric (TE) transmissions at $50^{\circ}$ separately. When fitting such measured transmission spectra to the predictions from our transfer matrix model, shown both in Figure 2d, we observe a small IR shift for the predicted transmission relative to the measured one. However, this shift is present for both polarizations as well as for the normal incidence transmission. In such event, the small deviation observed should be attributed to a small error when determining each layer thickness or refractive index. Although we cannot fully discard it, if present, birefringence would be small enough not to introduce any significant change in the design of the 1D-NPS to obtain an optimal performance for the transparent solar cells.

\subsection{Efficiency in Energy Collection When Sunlight Is Incident at Large Angles}

As can be inferred from Figures $1 \mathrm{c}$ and 2a,b, the PCE for a five-layer 1D-NPS cell exhibits a strong dependence on the light angle of incidence. Indeed, if we compare the measured average PCEs from Table 1 we note that such average PCE increases by $13 \%$ when the cell is tilted from $0^{\circ}$ to $50^{\circ}$. As seen 
Table 2. Energy collection for the standard and the five-layer ID-NPS solar cells and the reference mc-Si module during the spring equinox (cells were positioned vertically and facing south).

\begin{tabular}{|c|c|c|c|}
\hline Device & Accumulated electric energy $(E)\left[\mathrm{kWh} \mathrm{m}^{-2} \mathrm{~d}\right]$ & Solar radiation $\left(E_{\text {sun }}\right)\left[\mathrm{kWh} \mathrm{m}^{-2} \mathrm{~d}\right]$ & Energy conversion efficiency (ECE) [\%] \\
\hline Standard $\left.{ }^{\mathrm{a}, \mathrm{b}}\right)$ & $0.268 \pm 0.010(0.276)$ & 5.092 & $5.26 \pm 0.20(5.42)$ \\
\hline Five-layer 1D-NPS ${ }^{a)}$ & $0.554 \pm 0.011(0.570)$ & 5.825 & $9.51 \pm 0.19(9.80)$ \\
\hline $\mathrm{mc}-\mathrm{Si}$ & 0.723 & 5.825 & 12.42 \\
\hline
\end{tabular}

a) The average values and standard deviations are obtained from 16 devices and the highest values are shown in parentheses; b) Measured on a different day (4 days after the equinox).

in Figure 1c, PCE tends to maximize close to the Brewster angle and approaches $0^{\circ}$ at $90^{\circ}$. As indicated above for an optimal cell architecture, the inverse design must be set to maximize ECE during the course of 1 day rather than PCE at a given angle. This should properly account for PCE as well as incident intensity dependence on the angle of incidence or local solar time. As seen in Figure 3a, during or close to the equinox for any flat device positioned vertically and facing south, the angle of incidence for sun rays will range from $48^{\circ}$ to $90^{\circ}$, while sunlight intensity will range from 0 to around $800 \mathrm{~W} \mathrm{~m}^{-2}$. We have chosen to perform outdoor measurements during or close to the equinox because they provide a good average over year variations in sun rays intensity and altitude.

The electric energy accumulated during a day (in $\mathrm{kWh} \mathrm{m}^{-2}$ ) as well as the energy conversion efficiency (following Equation S13, Supporting Information) is determined per square meter of installation or relative to the cell area. As shown in Figure 3b and Table 2, the five-layer 1D-NPS can collect energy with an average efficiency of $9.51 \%$, which is slightly larger than $9.40 \%$, its PCE at $50^{\circ}$ incidence. As can be seen by inspection of Figure 3c, a similar relation between PCE and ECE is found for the multi-crystalline silicon (mc-Si) module incorporating a light diffusion texturing. On the contrary, the standard configuration transparent OPV cell exhibits an average $5.26 \%$ ECE, a $13 \%$ less relative to $6.02 \%$, its PCE at $50^{\circ}$ incidence. In the inverse design to optically tailor the 1D-NPS cell architecture, the computed and measured current densities (shown in Figure $3 \mathrm{~d}$ ) are relative to the actual cell area to account for the reduction in the total number of collected photons by the $\cos \alpha$ factor. ${ }^{[43-45]}$ In addition, one may also introduce the $F F \cdot V_{\mathrm{OC}}$ product variations due to changes in sunlight intensity during the course of 1 day, shown in Figure $3 \mathrm{~d}$. When the latter and all other light dependent effects are taken into account, the inverse design yields a cell architecture for the most efficient energy conversion. As can be seen in Figure 3c, the PCE for the 1D-NPS cell is for a wide hourly range fairly constant, exhibiting a slight increase around 8 and $16 \mathrm{~h}$ and a clear drop during very early or late hours.

\section{Conclusion}

The optical tailoring brought by the 1D-NPS is shown to provide an optimal sunlight collection at large angles of $\approx 50^{\circ}$ and above. For a broad range of angles $\left(\approx 20-80^{\circ}\right)$ the capacity of a highly transparent cell to convert light into electricity is increased by more than $50 \%$ relative to a transparent device in a standard configuration not including any kind of specific architecture to decouple light harvesting from transmission. When considering a vertical installation, we demonstrated that the PCE as well as the ECE during the equinox are both larger than 9.5\%. These efficiencies are the highest ever measured for any kind of PV technology exhibiting an AVT larger than $50 \%$. The efficient utilization of IR radiation by the blend, the small number of $\lambda / 4$ layers tuned at different wavelengths used in the optical tailoring, the energy harvesting decoupled from transmission, the enhanced harvesting at large angles of incidence, and the extremely thin metallic top electrode fabricated, all align together in the same direction to lead to an increase in light to electricity conversion larger than $50 \%$, as seen in Table 3, where three different metrics to evaluate a semitransparent cell are compared.

It is worth noting that vertical sunlight collection measurements of the ECE performed during the equinox provide a good average of the device performance throughout the year. At parallel $41^{\circ}$ during the spring and fall equinoxes, sun rays will be incident on the cell at angles that range from $48^{\circ}$ to $90^{\circ}$. The angular range is from $25^{\circ}$ to $60^{\circ}$ during the winter solstice and from $72^{\circ}$ to $90^{\circ}$ during the summer solstice. In addition, provided that diffused sunlight is mostly irradiated from the

Table 3. Performance of the 1D-NPS transparent cell compared.

\begin{tabular}{|c|c|c|c|c|c|c|c|c|}
\hline & & \multicolumn{2}{|c|}{ PCE [\%] } & \multicolumn{2}{|c|}{ PCE.AVT [\%] } & \multicolumn{3}{|c|}{ ECE [\%] } \\
\hline & & Standard $0^{\circ}$ & Standard $50^{\circ}$ & Standard $0^{\circ}$ & Standard $50^{\circ}$ & mc-Si ( $100 \%$ area) & mc-Si ( $50 \%$ area) & Standard \\
\hline \multirow{4}{*}{$\begin{array}{l}\tilde{n} \\
\sum_{1} \\
\underline{0}\end{array}$} & & 5.82 & 6.11 & 2.99 & 3.14 & 12.42 & 6.21 & 5.42 \\
\hline & $0^{\circ}$ & 8.66 & & 4.33 & & & & \\
\hline & $50^{\circ}$ & & 9.67 & & 4.83 & & & \\
\hline & All angles & & & & & 9.80 & 9.80 & 9.80 \\
\hline Gain (\%) & & 49 & 58 & 45 & 54 & -21 & 58 & 81 \\
\hline
\end{tabular}


circumsolar region, as shown in Figure S14, Section S6 (Supporting Information), one should not expect any significant drop in cell performance when such performance is averaged taking into account all possible weather and sky conditions as well as different sun altitudes and irradiances throughout the year.

It is interesting to establish a comparison of the 1D-NPS cells with commercial mc-Si modules. The nominal PCE of such modules is, in the newer models, close to $17 \%$, about 2 percentage points higher than the older mc-Si module we used as a reference to evaluate conversion to electrical energy during the course of a day (Figure 3b,c). In normal operating conditions, this efficiency would drop to $15 \%$, exhibiting a loss of 2 percentage points, similar to the loss we measured for the mc-Si module we tested. When considering a vertical Si installation covering $50 \%$ of a façade, in other words, in a chessboard like building integrated photovoltaic (BIPV) installation that would provide a $50 \%$ obstruction to light pass, the energy collection efficiency would drop to $7.5 \%$. On the other hand, assuming $10 \%$ losses in an eventual up-scaling to modules of the neutral color transparent OPV cells we fabricated, such OPV technology incorporating a three-layer 1D-NPS would exhibit approximately 7.5\% PCE or ECE. In summary, we can say that the optically tailored transparent OPV technology that we developed has the potential to clearly outperform commercial Si modules for vertical façade BIPV installations. It would provide a similar light to electricity conversion but with a high quality $50 \%$ transparency at normal incidence without any highly visible obstruction to vision or drastic change in color. Away from normal incidence and up to $50^{\circ}$, changes in transmission are minor as can be seen in Figure S10 (Supporting Information) where the transmission for a three-layer 1D-NPS was measured at angles ranging from $0^{\circ}$ to $50^{\circ}$. In addition, given that the human eye response to changes in light intensity is logarithmic, the $50 \%$ reduction in transparency of the OPV device corresponds to a loss in brightness perception of only $20 \%{ }^{[46,47]}$ (see Section S4, Supporting Information). Such loss in brightness is similar to the one obtained from technical glasses, which are widely used in transparent building façades. Similarly, other chromatic attributes ${ }^{[48]}$ of the transparent OPV devices (Table S5 and Figure S16, Supporting Information) indicate that color neutrality and device performance can be attained simultaneously. Finally, one must note that no significant cell degradation is observed when the encapsulated cells are kept in ambient conditions in the dark. However, future work should address cell performance evolution under illumination which, due to the presence of the NFA, seems to deviate significantly from the evolution observed in highly stable fullerene acceptor based cells. ${ }^{[49,50]}$

Regarding potential applications for an energy collection technology more efficient when light is incident at large angles, it is important to note that in many elements or structures where PV is likely to be integrated in, sunlight would in fact be incident at such large angles for most of the time. Window façades but also electric vehicles are two very relevant applications that may benefit from transparent photovoltaics. Uses for such technology as well as the optical tailoring developed may go beyond a simple light to electricity conversion. An interesting application may arise in two-photon absorption artificial photosynthesis systems with a tandem configuration. In such case, a transparent technology would allow for an optimal integration of such systems while the optical tailoring would lead to a better balance among the n-type semiconductor photoanode and solar cell currents. Both issues may trigger a major step forward in solar fuel production research.

\section{Experimental Section}

Transparent Cell Fabrication: First, a $\approx 10 \mathrm{~nm} \quad \mathrm{ZnO}$ electrontransporting layer was deposited on a precleaned ITO substrate from a sol-gel precursor solution of zinc acetate dehydrate dissolved in 2-methoxyethanol $(0.15 \mathrm{M}) \cdot{ }^{[51,52]}$ After thermally annealing at $150{ }^{\circ} \mathrm{C}$ in air (relative humidity < 40\%) for $20 \mathrm{~min}$, the $\mathrm{ZnO}$-coated samples were transferred into a nitrogen-filled glovebox for spin-casting the PTB7Th:FOIC:PC ${ }_{71}$ BM (1:0.75: 0.75 by weight) photoactive layer. ${ }^{[29]}$ Ternary blend solutions were deposited at different concentrations and spinning speeds to achieve the numerically computed active layer thicknesses, i.e., $A L$ thickness of $\approx 50, \approx 70$, and $\approx 86 \mathrm{~nm}$ were achieved with $15,17.5$, and $20 \mathrm{mg} \mathrm{mL}^{-1}$ solutions (total concentration), respectively. Afterwards, the resulting blend films were dried under vacuum $\left(<5 \times 10^{-6}\right.$ mbar $)$ for $1 \mathrm{~h}$. Finally, a $5 \mathrm{~nm}$ thick $\mathrm{MoO}_{3}$ layer (Alfa Aesar, $0.5 \AA \mathrm{s}^{-1}$ ) followed by an ultrathin $A u$ seed layer $\left(0.4 \AA \mathrm{s}^{-1}, \approx 1 \mathrm{~nm}\right)$ and a thin $\mathrm{Ag}$ top electrode (3 $\AA \mathrm{s}^{-1}, 8-14 \mathrm{~nm}$ ) were deposited sequentially through a shadow mask by thermal evaporation $\left(<5 \times 10^{-6} \mathrm{mbar}\right)$. The device area, defined by the overlap between ITO and top thin Ag electrodes, was $0.06 \mathrm{~cm}^{2}$. Note that the evaporation of the $\mathrm{Au} / \mathrm{Ag}$ thin electrode was carried out on a substrate cooled down to $-5{ }^{\circ} \mathrm{C}$ in order to decrease surface diffusion and island formation of the $\mathrm{Ag}$ film. The optical constant for the thin Ag films (Figure S6a, Supporting Information) was accurately extracted through fitting experimental light transmission, yielding an excellent agreement with the experimental results (Figure S6b, Supporting Information). For standard cells, an additional protecting layer of LiF $\left(0.5 \AA \mathrm{s}^{-1}, 30 \mathrm{~nm}\right)$ was deposited on top of the device area. Finally, to ensure good electrical contact, an additional $100 \mathrm{~nm}$ thick Ag film was deposited on top of the outer edge of the thin Ag electrode.

1D-NPS and ARC Deposition: The 1D-NPS were fabricated by sequential thermal evaporation of relatively low melting-point $\mathrm{MoO}_{3}$ and LiF inorganic materials. To avoid introducing physical damage or electrical degradation of the cell underneath, and to guarantee batchto-batch reproducibility, the deposition rates for both materials need to be well controlled at $0.5-0.6 \AA \mathrm{s}^{-1}$. The thicknesses of all 1D-NPS layers were carefully monitored during the deposition using a crystal quartz balance, and were later confirmed by fitting the measured transmission curves with a light transmission model. For the three-layer and five-layer 1D-NPS cells, an additional $120 \mathrm{~nm}$ LiF ARC layer was thermally evaporated on the front side of the substrates. All indoor cell measurements were performed in ambient air without encapsulation, whereas all outdoor measurements were done on glass-encapsulated devices sealed with a UV curable adhesive (Norland Optical Adhesive $73,1.5$ min curing) before testing. A picture of one of the encapsulated devices used for the outdoor measurements is shown in Figure S11a (Supporting Information). Note that after encapsulation, the five-layer 1D-NPS cell exhibits negligible changes in EQE, as seen in Figure S12 (Supporting Information).

Characterization: The optical simulated transmissions and EQEs at normal incidence and higher incidence angles were performed using an in-house computer code based on the transfer matrix method. The required optical constants (refractive index and extinction coefficient) of all materials used in the devices, shown in Figure S17 (Supporting Information), were obtained from modeling the experimental transmission of the thin films for various thicknesses, unless stated otherwise. The $J-V$ curves of all devices were measured with a Keithley 2420 source meter under a 1-sun, AM1.5G spectrum from a solar simulator (ABET Sol3A, $1000 \mathrm{~W} \mathrm{~m}^{-2}$ ). The illumination intensity of the light source (Xenon lamp, 300W, USHIO) was calibrated using a 
Hamamatsu silicon photodiode (with a KG-5 filter, area $=0.1296 \mathrm{~cm}^{2}$ ) certificated by ISE Fraunhofer. Spectrally resolved EQE responses were measured using a benchtop equipment (QEX10, PV Measurements Inc) at $130 \mathrm{~Hz}$, using a calibrated silicon cell spectral response as reference. For the $J-V$ and $E Q E$ measurements at large incidence angles, an in-house sample holder with tunable tilt was employed. For the EQE measurements, special care was taken to guarantee that the light spot $\left(3 \mathrm{~mm}\right.$ in length at $\left.0^{\circ}\right)$ was completely enclosed within the active cell area. The light transmission spectra for the fabricated devices were recorded using an UV-VIS-NIR spectrophotometer (Lambda950, PerkinElmer). Similarly, the beam spot was completely confined within the device area. TE and TM polarized light transmissions for fivelayer 1D-NPS cell at normal incidence and $50^{\circ}$ were recorded by a spectroscopic ellipsometer (Sopra, GES-5E). The sheet resistance of top thin $\mathrm{Ag}$ electrodes was determined by a four-point probe station (Cascade Microtech 44/7) connected to a Keithley 2001 multimeter. The morphological characteristics of thin-Ag films were studied by field emission scanning electron microscopy (FEG-SEM, FEI Inspect F-EBL).

Direct Sunlight Measurements: The outdoor monitoring station was located on ICFO's roof (latitude: $41.27^{\circ}$; longitude: $1.989^{\circ}$ ), with a vertical support oriented toward solar south and free of any shadows and highly reflective surfaces. The irradiance sensor was a thermopile pyranometer (Apogee Instruments SP-500 Series) with a certified calibration uncertainty of $\pm 5 \%$ (ISO-classified reference). The mc-Si module (REC Group) had a rated peak power of $20 \mathrm{~W}$ and $\approx 0.135 \mathrm{~m}^{2}$ active area, with a resistance temperature detector (PT100) adhered to the back panel. A photograph and a system schematic is shown in Figure S11 (Supporting Information). The J-V curves of the encapsulated test devices and the mc-Si module were measured with a Keithley $2635 \mathrm{~A}$ source meter (4-wire probing) at $1 / 4-1 \mathrm{~h}$ intervals using a commercial data logging software (Tracer IV, ReRa solutions). Measurements were done during or near the spring equinox (March 21), ensuring cloudless conditions were present trying to minimize the contribution of diffuse irradiance. A black surface was used on the back of the cells to prevent rear reflections. Because the cells were only exposed to sunlight during the voltage sweep in order to avoid light degradation and ensure data consistency, the possible effects of high operating temperatures are not considered, although it is generally accepted that PCE temperature coefficients in OPV are positive, improving the PCE values slightly. ${ }^{[33,54]}$ Moreover, the PCE was measured under a solar simulator before and after each outdoor run, showing no signs of degradation (see Figure S13, Supporting Information).

\section{Supporting Information}

Supporting Information is available from the Wiley Online Library or from the author.

\section{Acknowledgements}

Q.L., L.G., F.B., J.T., and J.M. acknowledge the financial support from the Spanish Ministry of Economy and Competitiveness (MINECO) through the "Severo Ochoa" program for Centres of Excellence in R\&D (SEV2015-0522), from Fundació Privada Cellex, from Generalitat de Catalunya through the Impulsa-Llavor (Llav-00073) and CERCA programs, and also acknowledge financial support from MINECO through project MAT2017. 89522-R. T.L. and X.Z. thank the NSFC (No. 21734001). F.B. also thanks the CONACYT international grants program.

\section{Conflict of Interest}

The authors declare no conflict of interest.

\section{Keywords}

1-D nanophotonic structures, near-infrared ternary blends, oblique incidence, optical trapping, transparent solar cells

Received: December 21, 2019

Revised: February 6, 2020

Published online: March 13, 2020

[1] C. J. Traverse, R. Pandey, M. C. Barr, R. R. Lunt, Nat. Energy 2017, 2,849 .

[2] Q. Xue, R. Xia, C. J. Brabec, H. L. Yip, Energy Environ. Sci. 2018, 11, 1688.

[3] S. Dai, X. Zhan, Adv. Energy Mater. 2018, 8, 1800002.

[4] Y. Li, G. Xu, C. Cui, Y. Li, Adv. Energy Mater. 2018, 8, 1701791.

[5] F. Liu, Z. Zhou, C. Zhang, J. Zhang, Q. Hu, T. Vergote, F. Liu, T. P. Russell, X. Zhu, Adv. Mater. 2017, 29, 1606574.

[6] Y. Cui, C. Yang, H. Yao, J. Zhu, Y. Wang, G. Jia, F. Gao, J. Hou, Adv. Mater. 2017, 29, 1703080.

[7] W. Wang, C. Yan, T.-K. Lau, J. Wang, K. Liu, Y. Fan, X. Lu, X. Zhan, Adv. Mater. 2017, 29, 1701308.

[8] Y. Li, J.-D. Lin, X. Che, Y. Qu, F. Liu, L.-S. Liao, S. R. Forrest, J. Am. Chem. Soc. 2017, 139, 17114.

[9] T. Li, S. Dai, Z. Ke, L. Yang, J. Wang, C. Yan, W. Ma, X. Zhan, Adv. Mater. 2018, 30, 1705969.

[10] S. Chen, H. Yao, B. Hu, G. Zhang, L. Arunagiri, L.-K. Ma, J. Huang, J. Zhang, Z. Zhu, F. Bai, W. Ma, H. Yan, Adv. Energy Mater. 2018, 8, 1800529.

[11] B. Jia, S. Dai, Z. Ke, C. Yan, W. Ma, X. Zhan, Chem. Mater. 2018, 30, 239.

[12] H. Huang, X. Li, L. Zhong, B. Qiu, Y. Yang, Z.-G. Zhang, Y. Li, J. Mater. Chem. A 2018, 6, 4670.

[13] J. Zhang, G. Xu, F. Tao, G. Zeng, M. Zhang, Y. Yang, Y. Li, Y. Li, Adv. Mater. 2019, 31, 1807159.

[14] J. Wang, J. Zhang, Y. Xiao, T. Xiao, R. Zhu, C. Yan, Y. Fu, G. Lu, X. Lu, S. R. Marder, X. Zhan, J. Am. Chem. Soc. 2018, 140, 9140.

[15] J. Zhu, Y. Xiao, J. Wang, K. Liu, H. Jiang, Y. Lin, X. Lu, X. Zhan, Chem. Mater. 2018, 30, 4150.

[16] M. B. Upama, M. Wright, N. K. Elumalai, M. A. Mahmud, D. Wang, C. Xu, A. Uddin, ACS Photonics 2017, 4, 2327.

[17] Y. Liu, P. Cheng, T. Li, R. Wang, Y. Li, S.-Y. Chang, Y. Zhu, H.-W. Cheng, K.-H. Wei, X. Zhan, B. Sun, Y. Yang, ACS Nano 2019, $13,1071$.

[18] X. Ma, Z. Xiao, Q. An, M. Zhang, Z. Hu, J. Wang, L. Ding, F. Zhang, J. Mater. Chem. A 2018, 6, 21485.

[19] H. Shi, R. Xia, G. Zhang, H.-L. Yip, Y. Cao, Adv. Energy Mater. 2019, 9, 1803438

[20] W. Yu, L. Shen, P. Shen, Y. Long, H. Sun, W. Chen, S. Ruan, ACS Appl. Mater. Interfaces 2014, 6, 599.

[21] W. Yu, S. Ruan, Y. Long, L. Shen, W. Guo, W. Chen, Sol. Energy Mater. Sol. Cells 2014, 127, 27.

[22] C. Bronnbauer, J. Hornich, N. Gasparini, F. Guo, B. Hartmeier, N. A. Luechinger, C. Pflaum, C. J. Brabec, K. Forberich, Adv. Opt. Mater. 2015, 3, 1424.

[23] Y. Zhang, Z. Peng, C. Cai, Z. Liu, Y. Lin, W. Zheng, J. Yang, L. Hou, Y. Cao, J. Mater. Chem. A 2016, 4, 11821.

[24] P. Shen, M. Yao, J. Liu, Y. Long, W. Guo, L. Shen, J. Mater. Chem. A 2019, 7, 4102

[25] G. Xu, L. Shen, C. Cui, S. Wen, R. Xue, W. Chen, H. Chen, J. Zhang, H. Li, Y. Li, Y. Li, Adv. Funct. Mater. 2017, 27, 1605908.

[26] J. Zhong, W. Liang, Y. Long, Optik 2018, 171, 706.

[27] V. Ganapati, O. D. Miller, E. Yablonovitch, IEEEJ. Photovoltaics 2014, 4, 175.

[28] S. Molesky, Z. Lin, A. Y. Piggott, W. Jin, J. Vucković, A. W. Rodriguez, Nat. Photonics 2018, 12, 659. 
[29] Q. Liu, J. Toudert, T. Li, M. Kramarenko, G. Martínez-Denegri, L. Ciammaruchi, X. Zhan, J. Martorell, Adv. Energy Mater. 2019, 9, 1900463.

[30] R. Xia, C. J. Brabec, H. L. Yip, Y. Cao, Joule 2019, 3, 2241.

[31] Y. Li, C. Ji, Y. Qu, X. Huang, S. Hou, C.-Z. Li, L.-S. Liao, L. J. Guo, S. R. Forrest, Adv. Mater. 2019, 31, 1903173.

[32] R. Betancur, P. Romero-Gomez, A. Martinez-Otero, X. Elias, M. Maymó, J. Martorell, Nat. Photonics 2013, 7, 995.

[33] F. Pastorelli, P. Romero-Gomez, R. Betancur, A. Martinez-Otero, P. Mantilla-Perez, N. Bonod, J. Martorell, Adv. Energy Mater. 2015, 5, 1400614

[34] H. Zhang, M. Kramarenko, J. Osmond, J. Toudert, J. Martorell, ACS Photonics 2018, 5, 2243.

[35] C.-Y. Chang, L. Zuo, H.-L. Yip, Y. Li, C.-Z. Li, C.-S. Hsu, Y.-J. Cheng, H. Chen, A. K.-Y. Jen, Adv. Funct. Mater. 2013, 23, 5084.

[36] H. Shi, R. Xia, C. Sun, J. Xiao, Z. Wu, F. Huang, H.-L. Yip, Y. Cao, Adv. Energy Mater. 2017, 7, 1701121.

[37] J. Yun, Adv. Funct. Mater. 2017, 27, 1606641.

[38] N. P. Sergeant, A. Hadipour, B. Niesen, D. Cheyns, P. Heremans, P. Peumans, B. P. Rand, Adv. Mater. 2012, 24, 728.

[39] S. Schubert, J. Meiss, L. Müller-Meskamp, K. Leo, Adv. Energy Mater. 2013, 3, 438.

[40] P. Romero-Gomez, R. Betancur, A. Martinez-Otero, X. Elias, M. Mariano, B. Romero, B. Arredondo, R. Vergaz, J. Martorell, Sol. Energy Mater. Sol. Cells 2015, 137, 44.
[41] T. Stefaniuk, P. Wróbel, E. Górecka, T. Szoplik, Nanoscale Res. Lett. 2014, 9, 153

[42] J. Bergqvist, H. Arwin, O. Inganäs, ACS Photonics 2018, 5, 3023.

[43] J. Mescher, A. Mertens, A. Egel, S. W. Kettlitz, U. Lemmer, A. Colsmann, AIP Adv. 2015, 5, 077188.

[44] D. Cheyns, B. P. Rand, B. Verreet, J. Genoe, J. Poortmans, P. Heremans, Appl. Phys. Lett. 2008, 92, 243310.

[45] G. Dennler, K. Forberich, M. C. Scharber, C. J. Brabec, I. Tomiš, K. Hingerl, T. Fromherz, J. Appl. Phys. 2007, 102, 054516.

[46] S. T. Stevens, Science 1961, 133, 80.

[47] B. Bauer, Psychol. Record 2009, 59, 171.

[48] C. Yang, D. Liu, M. Bates, M. C. Barr, R. R. Lunt, Joule 2019, 3, 1803.

[49] Q. Liu, J. Toudert, F. Liu, P. Mantilla-Perez, M. M. Bajo, T. P. Russell, J. Martorell, Adv. Energy Mater. 2017, 7, 1701201.

[50] N. Y. Doumon, M. V. Dryzhov, F. V. Houard, V. M. Le Corre, A. R. Chatri, P. Christodoulis, L. J. A. Koster, ACS Appl. Mater. Interfaces 2019, 11, 8310.

[51] Q. Liu, P. Romero-Gomez, P. Mantilla-Perez, S. Colodrero, J. Toudert, J. Martorell, Adv. Energy Mater. 2017, 7, 1700356.

[52] Q. Liu, J. Toudert, L. Ciammaruchi, G. Martínez-Denegri, I. Martorell, J. Mater. Chem. A 2017, 5, 25476.

[53] N. Bristow, J. Kettle, J. Renewable Sustainable Energy 2015, 7, 013111.

[54] I. Riedel, J. Parisi, V. Dyakonov, L. Lutsen, D. Vanderzande, J. C. Hummelen, Adv. Funct. Mater. 2004, 14, 38. 